\title{
Clinical Characteristics and Prognosis of Patients with COVID-19 Combineded with or without Diabetes, Hypertension or Coronary
}

Haoxiang Li

Affiliated Hospital of Jiangsu University

Jianguo Zhang

Affiliated Hospital of Jiangsu University

Jinhui Zhang

Affiliated Hospital of Jiangsu University

Ling Yang

Affiliated Hospital of Jiangsu University

Dong Wang

Affiliated Hospital of Jiangsu University

Li Zhao

Affiliated Hospital of Jiangsu University

Xia Deng

Affiliated Hospital of Jiangsu University

Guoyue Yuan (D yuanguoyue08@163.com )

Original investigation

Keywords: COVID-19, diabetes, hypertension, coronary

Posted Date: June 30th, 2020

DOl: https://doi.org/10.21203/rs.3.rs-36840/v1

License: (c) (i) This work is licensed under a Creative Commons Attribution 4.0 International License.

Read Full License 


\section{Clinical Characteristics and Prognosis of patients with COVID-19 combineded}

with or without diabetes, hypertension or coronary

Haoxiang Li $^{1}$, Jianguo Zhang ${ }^{2}$, Jinhui Zhang ${ }^{2}$, Ling Yang ${ }^{1}$, Dong Wang ${ }^{1}$, Li Zhao ${ }^{1}$, Xia Deng ${ }^{1}$, Guoyue Yuan ${ }^{1}$

(1. Department of Endorcrinology, 2. Department of Intensive Care Unit, Affiliated Hospital of Jiangsu University, 438, Jiefang Road, Zhenjiang, Jiangsu 212001, China )

\section{corresponding author:}

Guoyue Yuan, M.D, PhD,

Department of Endocrinology, Affiliated Hospital of Jiangsu University, 438, Jiefang Road, Zhenjiang, Jiangsu 212001, China

Mobile: +8613505289352, Fax number: 86-511-85019237

Email: yuanguoyue@hotmail.com, yuanguoyue08@163.com 


\section{Abstract}

Bcakground: This study was to investigate the clinical characteristics and prognosis of COVID-19 patients combined with or without major chronic diseases like diabetes, hypertension or coronary.

Methods: We retrospectively analyzed 183 patients with COVID-19 diagnosed at First People's Hospital of Jiangxia District (FPHJD) in Wuhan, China attended by Affiliated Hospital of Jiangsu University supporting medical team from February 1, 2020 to March 15, 2020. Patients were divided into simple COVID-19 group(n=134), COVID-19 combined with diabetes, hypertension or coronary group( $\mathrm{n}=49)$. Besides, COVID-19 patients with diabetes, hypertension or coronary were further classified into severe pneumonia group $(n=23)$ and common pneumonia group $(n=26)$, death group $(n=17)$ and survival group $(n=32)$. The prognosis of COVID-19 patients was evaluated by analyzing the clinical data and the results of laboratory tests.

Results: 183 patients were included in this study, of whom 166 were discharged and 16 died in hospital. 49 (26.92\%) patients had a comorbidity, with hypertension being the most common [37 (20.33\%) patients], followed by diabetes [25 (13.74\%) patients] and coronary heart disease [4 (2.2\%) patients]. Compared with simple COVID-19 group, the proportion of history of chronic respiratory system disease, age, D-dimer, procalcitonin, C-reactive protein, myoglobin, cardiac troponin I, creatine kinase MB, lactate dehydrogenase, white blood cell count, neutrophil count, neutrophil percentage, blood urea nitrogen, creatinine and mortality rate were significantly higher in COVID-19 combined with chronic diseases group, whereas lymphocyte count, lymphocyte percentage and alanine transferase were significantly lower in COVID-19 combined with chronic diseases group. Among COVID-19 patients with chronic diseases, D-dimer, procalcitonin, C-reactive protein, myoglobin, cardiac troponin I, lactate dehydrogenase, white blood cell count, neutrophil count, neutrophil percentage, blood urea nitrogen, death rate was significantly higher in severe pneumonia group than common pneumonia group. While lymphocyte count and lymphocyte percentage were significantly lower in severe pneumonia group than common pneumonia group. Besides, we found that the proportion of history of chronic respiratory system disease, 
D-dimer, procalcitonin, myoglobin, cardiac troponin I, creatine kinase MB, lactate dehydrogenase, neutrophil count, neutrophil percentage, blood urea nitrogen were significantly higher in death group compared with survival group, whereas lymphocyte count and lymphocyte percentage were significantly lower in survival group. In COVID-19 combined with chronic diseases group, univariate logistic regression showed that the risk for severe pneumonia were D-dimer, C-reactive protein, lactate dehydrogenase, white blood cell count, neutrophil count and neutrophil percentage. Univariate logistic regression also showed that the risk for death were D-dimer, lactate dehydrogenase, white blood cell count, neutrophil count, neutrophil percentage and blood urea nitrogen. Multivariate regression logistic showed that lactate dehydrogenase were independent risk factors for death among COVID-19 patients combined with chronic diseases. Cox regression analysis showed that compared with simple COVID-19 group, the RR(95\% CI) in COVID-19 patients combined with diabetes, hypertension, and coronary were 2.187 (1.141 4.191) for death $(P<0.05)$.

Conclusion: Among COVID-19 patients combined with diabetes, hypertension or coronary, the risk factors for severe pneumonia were D-dimer, C-reactive protein, lactate dehydrogenase, white blood cell count, neutrophil count and neutrophil percentage, whereas the risk factors for death were D-dimer, lactate dehydrogenase, white blood cell count, neutrophil count, neutrophil percentage and blood urea nitrogen. Moreover, lactate dehydrogenase were independent risk factors for death. The mortality rate of COVID-19 patients combined with diabetes, hypertension or coronary was higher than that of simple COVID-19 patients.

Key words: COVID-19; diabetes; hypertension; coronary 
Background: Coronavirus Disease 2019 (COVID-19) has quickly progressed to a global health emergency. COVID-19 is a new type of coronavirus which belongs to the Betacoronavirus genera with the capacity for rapid mutation and recombination.[1]. The main clinical symptoms of COVID-19 are fever, fatigue, and a dry cough, and in severe cases, multiple organ failure[2-3]. COVID-19 has numerous transmission channels and humans are highly susceptible to infection. Cases of COVID-19 have been found across the whole of China and overseas[4]. Recent studies have found that COVID-19 patients with chronic diseases like diabetes, hypertension and coronary have higher critical illness rate and mortality rate[5-6]. However, the risk factors for mortality and a detailed clinical course of illness have not been well described. This paper reviews and summarizes the epidemiological and clinical characteristics of COVID-19 patients combined with or without diabetes, hypertension or coronary in order to provide a reliable basis for early diagnosis and treatment.

\section{Methods}

\section{Study population}

This retrospective cohort study included patients with COVID-19 diagnosed at the First People's Hospital of Jiangxia District (FPHJD) in Wuhan, China attended by Affiliated Hospital of Jiangsu University supporting medical team from February 1, 2020 to March 15, 2020. In this study, patients who were hospitalised for COVID-19 and had a definite outcome (dead or discharged) at the early stage of the outbreak. The study was approved by the Research Ethics Commission of FPHJD (Approval No. 2020021) and written consent was waived by Ethics Commission of FPHJD.

\section{Data collection}

After taking medical history, necessary investigations like blood examinations which included complete blood count, serum biochemical tests (including renal and liver function, creatine kinase, creatine kinase MB and lactate dehydrogenase), myocardial enzymes(including myoglobin and cardiac troponin I), D-dimer, C-reactive protein 
and procalcitonin were performed upon hospital admission. All data were checked by two physicians (JZ and JZ).

\section{Definitions}

All patients were up to 18 years old and received throat swab samples which were gathered for SARS-CoV-2 RNA detection by gene sequencing or real-time RT-PCR and the results were positive at least once. Diabetes mellitus was defined as a medical history of diabetes or the use of oral hypoglycemic medication or insulin or patients with a fasting glucose $\geq 7.0 \mathrm{mmol} / \mathrm{L}$ or a two-hour postprandial serum glucose $\geq 11.1$ $\mathrm{mmol} / \mathrm{L}[7]$. Hypertension was defined as a medical history of hypertension or the use of antihypertensive, a systolic blood pressure of $\geq 140 \mathrm{mmHg}$ and/or a diastolic blood pressure of $\geq 90 \mathrm{mmHg}[8]$. Coronary diagnosis was defined as ischemic symptoms or coronary computed tomographic angiography (CTA) or percutaneous coronary interve ntion (PCI) and defined as angiographic evidence of more than $50 \%$ luminal narrowing in at least one segment of a main epicardial coronary artery[9]. The illness severity of pneumonia was defined according to the Chinese management guideline for COVID-19 (version 6.0)[10]. All the subjects were exclude from malignancy, pregnancy, blood disease, autoimmune disease and patients who failed to complete relevant blood examinations.

\section{Statistical Analysis}

All statistical analyses were performed using SPSS version16.0 (SPSS Inc., Chicago, IL). Data were summarized as means \pm standard deviations for normally distributed variables, medians plus percentiles $\left(25^{\text {th }} ; 7^{\text {th }}\right)$ for nonnormally distributed variables, and frequencies for categorical variables. For comparisons between two groups, independent Student $\mathrm{t}$ test was used. Categorical variables were examined by $\mathrm{x}^{2}$ test. To explore the risk factors associated with severe pneumonia and death, univariable and multivariable logistic regression models were used. To identify the chronic diseases affecting the mortality rate, we used a multivariate Cox regression analysis 
model. All calculated $p$ values were two-sided, and $p$ values $<0.05$ were considered statistically significant.

\section{Results}

Clinical and biochemical characteristics between simple COVID-19 group and COVID-19 combined with chronic diseases group

Compared with simple COVID-19 group, the proportion of history of chronic respiratory system disease, age, D-dimer, procalcitonin, C-reactive protein, myoglobin, cardiac troponin I, creatine kinase MB, lactate dehydrogenase, white blood cell count, neutrophil count, neutrophil percentage, blood urea nitrogen, creatinine and mortality rate were significantly higher in COVID-19 combined with chronic diseases group, whereas lymphocyte count, lymphocyte percentage and alanine transferase were significantly lower in COVID-19 combined with chronic diseases group(Table 1).

Table 1: Clinical and biochemical findings of patients on admission

\begin{tabular}{|c|c|c|c|}
\hline & $\begin{array}{c}\text { simple COVID-19 group } \\
(\mathrm{n}=134)\end{array}$ & $\begin{array}{l}\text { COVID-19 combined with } \\
\text { chronic diseases group }(n=49)\end{array}$ & $P$ value \\
\hline $\operatorname{Sex}(F / M)$ & $65 / 69$ & $30 / 19$ & 0.127 \\
\hline $\begin{array}{l}\text { history of chronic respiratory } \\
\text { system disease(yes/no) }\end{array}$ & $1 / 134$ & $3 / 46$ & $0.022 *$ \\
\hline Age(year) & $50.37 \pm 16.45$ & $66.49 \pm 12.24$ & $<0.001 *$ \\
\hline D-dimer, mg/L & $0.37(0.19,0.84)$ & $0.67(1.26,1.89)$ & $0.009^{*}$ \\
\hline Procalcitonin, ng/ml & $0.06(0.04,0.13)$ & $0.10(0.05,0.25)$ & $0.005 *$ \\
\hline C-reactive protein, $\mathrm{mg} / \mathrm{L}$ & $10.39(1.16,46.96)$ & $40.49(12.93,85.15)$ & $0.001 *$ \\
\hline myoglobin, $\mathrm{U} / \mathrm{L}$ & $25.82(21.00,55.43)$ & $49.37(25.60,217.25)$ & $<0.001 *$ \\
\hline cardiac troponin $\mathrm{I}, \mu \mathrm{g} / \mathrm{L}$ & $0.01(0.01,0.01)$ & $0.02(0.01,0.03)$ & $<0.001^{*}$ \\
\hline creatine kinase $\mathrm{MB}$ & $13.55(10.38,17.23)$ & $15.9(11.10 .26 .25)$ & 0.064 \\
\hline creatine kinase, $\mathrm{U} / \mathrm{L}$ & $72.60(44.50,122.00)$ & $77(50.50, \quad 146.00)$ & 0.668 \\
\hline lactate dehydrogenase, $\mathrm{U} / \mathrm{L}$ & $215.30(179.50,333.75)$ & 266(209.30,394.70) & $0.009 *$ \\
\hline white blood cell count, $\times 10^{\wedge} 9 / \mathrm{L}$ & $6.20(4.40,8.40)$ & $7.60(5.30,11.95)$ & $0.007^{*}$ \\
\hline neutrophil count, $\times 10^{\wedge} 9 / \mathrm{L}$ & $4.44(2.75,6.32)$ & $5.89(4.07,10.54)$ & $0.001^{*}$ \\
\hline neutrophil percentage, $\%$ & $70.59 \pm 13.99$ & $80.27 \pm 9.52$ & $<0.001^{*}$ \\
\hline lymphocyte count, $\times 10^{\wedge} 9 / \mathrm{L}$ & $1.06(0.71,1.58)$ & $0.73(0.55,1.19)$ & $0.003 *$ \\
\hline lymphocyte percentage, $\%$ & $17.75(10.48,30.15)$ & $12.20(6.87,16.55)$ & $<0.001 *$ \\
\hline platelet count, $\times 10^{\wedge} 9 / \mathrm{L}$ & $210.00(150.75,260.50)$ & $179.00(122.00,286.50)$ & 0.11 \\
\hline
\end{tabular}




\begin{tabular}{lccl} 
aspartate aminotransferase, $\mathrm{U} / \mathrm{L}$ & $26.60(19.43,38.88)$ & $26.50(20.40,38.85)$ & 0.857 \\
alanine aminotransferase, $\mathrm{U} / \mathrm{L}$ & $22.80(15.20,42.18)$ & $18.50(12.60,31.80)$ & $0.032^{*}$ \\
blood urea nitrogen, mmol/L & $4.45(3.40,5.50)$ & $5.80(4.50,11.05)$ & $<0.001^{*}$ \\
Creatinine, $\mu \mathrm{mol} / \mathrm{L}$ & $63.35(51.88,81.05)$ & $73.60(60.60,104.50)$ & $0.001^{*}$ \\
mortality rate & $21 / 134$ & $16 / 49$ & $<0.001^{*}$ \\
\hline
\end{tabular}

F: female; M: male.

Data are means $\pm \mathrm{SD}, \mathrm{n}$, and median $\left(25^{\text {th }}\right.$ and $75^{\text {th }}$ percentiles $) *{ }^{*} P<0.05$.

\section{Clinical and biochemical characteristics among COVID-19 patients with chronic diseases}

Among COVID-19 patients with chronic diseases, D-dimer, procalcitonin, C-reactive protein, myoglobin, cardiac troponin I, lactate dehydrogenase, white blood cell count, neutrophil count, neutrophil percentage, blood urea nitrogen and mortality rate were significantly higher in severe pneumonia group than common pneumonia group. While lymphocyte count and lymphocyte percentage were significantly lower in severe pneumonia group than common pneumonia group (Table 2). Besides, we found that the proportion of history of chronic respiratory system disease, D-dimer, procalcitonin, myoglobin, cardiac troponin I, creatine kinase $\mathrm{MB}$, lactate dehydrogenase, neutrophil count, neutrophil percentage, blood urea nitrogenwere significantly higher in death group compared with survival group, whereas lymphocyte count and lymphocyte percentage were significantly lower in survival group (Table 3 ).

Table 2: Clinical and biochemical findings between common pneumonia group and severe pneumonia group

\begin{tabular}{lccl}
\hline & $\begin{array}{c}\text { common pneumonia } \\
\text { group }(\mathbf{n = 2 6})\end{array}$ & $\begin{array}{c}\text { severe pneumonia group } \\
(\mathbf{n = 2 3})\end{array}$ & P value \\
\hline Sex(F/M) & $14 / 12$ & $16 / 7$ & 0.26 \\
history of chronic respiratory & $0 / 26$ & $3 / 20$ & 0.057 \\
system disease(yes/no) & & & \\
Age(year) & $65.19 \pm 11.48$ & $67.96 \pm 13.15$ & 0.436 \\
D-dimer, mg/L & $0.38(0.20,0.68)$ & $1.87(0.78,7.33)$ & $<0.001^{*}$ \\
Procalcitonin, ng/ml & $0.06(0.03,0.14)$ & $0.16(0.10,0.46)$ & $<0.001^{*}$
\end{tabular}


C-reactive protein, $\mathrm{mg} / \mathrm{L}$

myoglobin, $\mathrm{U} / \mathrm{L}$

cardiac troponin $\mathrm{I}, \mu \mathrm{g} / \mathrm{L}$

creatine kinase $\mathrm{MB}$

creatine kinase, $\mathrm{U} / \mathrm{L}$

lactate dehydrogenase, $\mathrm{U} / \mathrm{L}$

white blood cell count, $\times 10^{\wedge} 9 / \mathrm{L}$

neutrophil count, $\times 10^{\wedge} 9 / \mathrm{L}$

neutrophil percentage, $\%$

lymphocyte count, $\times 10^{\wedge} 9 / \mathrm{L}$

lymphocyte percentage, $\%$

platelet count, $\times 10^{\wedge} 9 / \mathrm{L}$

aspartate aminotransferase, $\mathrm{U} / \mathrm{L}$

alanine aminotransferase, $\mathrm{U} / \mathrm{L}$

blood urea nitrogen, $\mathrm{mmol} / \mathrm{L}$

Creatinine, $\mu \mathrm{mol} / \mathrm{L}$

mortality rate

F: female; M: male.

Data are means $\pm \mathrm{SD}, \mathrm{n}$, and median $\left(25^{\text {th }}\right.$ and $75^{\text {th }}$ percentiles $){ }^{*} P<0.05$.

Table 3: Clinical and biochemical findings between survival group and death

group

\begin{tabular}{lccc}
\hline & survival group $(\mathbf{n}=\mathbf{3 2})$ & death group $(\mathbf{n = 1 7})$ & $\boldsymbol{P}$ value \\
\hline Sex(F/M) & $19 / 13$ & $11 / 6$ & 0.715 \\
history of chronic respiratory & $0 / 32$ & $3 / 14$ & $0.014^{*}$ \\
system disease(yes/no) & & & \\
Age(year) & $66.22 \pm 12.25$ & $67.00 \pm 12.58$ & 0.834 \\
D-dimer, mg/L & $0.50(0.20,0.74)$ & $1.93(0.82,7.70)$ & $0.001^{*}$ \\
Procalcitonin, ng/ml & $0.07(0.04,0.18)$ & $0.15(0.09,0.47)$ & $0.015^{*}$ \\
C-reactive protein, mg/L & $30.16(4.70,89.75)$ & $58.99(28.59,81.11)$ & 0.118 \\
myoglobin, U/L & $40.43(23.70,117.20)$ & $149.00(31.97,373.30)$ & $0.049^{*}$ \\
cardiac troponin $\mathrm{I}, \mu \mathrm{g} / \mathrm{L}$ & $0.01(0.01,0.02)$ & $0.02(0.02,0.55)$ & $0.001^{*}$ \\
creatine kinase $\mathrm{MB}$ & $13.30(11.05,21.83)$ & $23.20(11.85,35.65)$ & $0.048^{*}$ \\
creatine kinase, $\mathrm{U} / \mathrm{L}$ & $74.50(49.75,137.00)$ & $86.00(47.00,167.00)$ & 0.698 \\
lactate dehydrogenase, $\mathrm{U} / \mathrm{L}$ & $248.00(208.95,280.03)$ & $493.00(244.00,627.50)$ & $0.002^{*}$ \\
white blood cell count, $\times 10^{\wedge} 9 / \mathrm{L}$ & $6.65(5.25,9.08)$ & $8.80(6.10,15.40)$ & 0.095 \\
neutrophil count, $\times 10^{\wedge} 9 / \mathrm{L}$ & $5.15(4.00,7.89)$ & $7.36(5.02,14.11)$ & $0.034^{*}$ \\
neutrophil percentage, $\%$ & $77.05 \pm 9.11$ & $86.32 \pm 7.19$ & $0.001^{*}$ \\
lymphocyte count, $\times 10^{\wedge} 9 / \mathrm{L}$ & $0.85(0.58,1.41)$ & $0.06(0.49,0.75)$ & $0.004^{*}$ \\
lymphocyte percentage, \% & $15.18 \pm 7.02$ & $7.56 \pm 4.37$ & $<0.001^{*}$
\end{tabular}

\begin{tabular}{cl}
$60.53(37.07,106.56)$ & $0.002^{*}$ \\
$126.50(26.89,276.70)$ & $0.044^{*}$ \\
$0.02(0.01,0.18)$ & $0.007^{*}$ \\
$17.10(10.60,34.60)$ & 0.218 \\
$92.00(53.00,157.00)$ & 0.352 \\
$446.40(226.00,611.00)$ & $<0.001^{*}$ \\
$9.10(4.80,14.90)$ & $0.044^{*}$ \\
$8.36(4.09,13.75)$ & $0.013^{*}$ \\
$86.23 \pm 6.95$ & $<0.001^{*}$ \\
$0.57(0.49,0.73)$ & $<0.001^{*}$ \\
$7.80 \pm 4.32$ & $<0.001^{*}$ \\
$61.00(110.00,233.00)$ & 0.087 \\
$28.60(20.30,44.60)$ & 0.435 \\
$16.60(11.60,33.90)$ & 0.561 \\
$7.60(5.50,13.10)$ & $0.014^{*}$ \\
$92.60(63.70,111.10)$ & 0.103 \\
$16 / 23$ & $<0.001^{*}$ \\
\hline
\end{tabular}

$191.00(141.00,310.00)$

$25.25(19.25,36.43)$

$5.20(4.15,8.13)$

68.60(55.83,97.93)

$0 / 26$

$16 / 23$

$<0.001 *$ 
platelet count, $\times 10^{\wedge} 9 / \mathrm{L}$

aspartate aminotransferase, U/L

alanine aminotransferase, $\mathrm{U} / \mathrm{L}$

blood urea nitrogen, $\mathrm{mmol} / \mathrm{L}$

Creatinine, $\mu \mathrm{mol} / \mathrm{L}$
$184.50(139.00,301.75)$

24.90(19.55,35.70)

$19.80(13.45,30.60)$

$5.30(4.23,9.88)$

$69.20(56.40,95.80)$
$146.00(102.00,212.00)$

0.106

$34.30(23.90,55.20)$

0.106

$16.60(12.10,16.20)$

0.975

$7.00(5.55,16.20)$

$0.02 *$

$96.10(62.55,138.10)$ 0.057

F: female; M: male.

Data are means $\pm \mathrm{SD}, \mathrm{n}(\%)$, and median $\left(25^{\text {th }}\right.$ and $75^{\text {th }}$ percentiles $) .{ }^{*} P<0.05$.

\section{Univariate and multivariate analysis of the risk factors for severe pneumonia and death in COVID-19 patients combined with chronic diseases}

The distribution of COVID-19 patients combined with diabetes, hypertension or coronary was showed in Table 4 . We calculated the individual comorbidity percentage in common pneumonia group and severe pneumonia group, survival group and death group, and plotted them versus each other as shown in Figure 1 and Figure 2. In COVID-19 combined with chronic diseases group, univariate logistic regression showed that the risk for severe pneumonia were D-dimer, C-reactive protein, lactate dehydrogenase, white blood cell count, neutrophil count and neutrophil percentage(Table 5). Univariate logistic regression also showed that the risk for death were D-dimer, lactate dehydrogenase, white blood cell count, neutrophil count, neutrophil percentage and blood urea nitrogen(Table 6). Multivariate logistic regression showed that lactate dehydrogenase were independent risk factors for death among COVID-19 patients combined with chronic diseases(Table 7).

Table 4: Distribution of COVID-19 patients combined with diabetes, hypertension or coronary

\begin{tabular}{lll}
\hline Items & Death group(n=16) & Suivival group(33) \\
\hline Diabetes & 3 & 9 \\
Hypertension & 4 & 18 \\
Coronary & 0 & 0 \\
Diabetes \& hypertension & 7 & 4 \\
Diabetes \& coronary & 0 & 0
\end{tabular}


Hypertension \& coronary

Diabetes \& Hypertension \& coronary

2

Table 5: Univariate logistic regression analysis of the risk factors for severe pneumonia in COVID-19 patients combined with chronic diseases

\begin{tabular}{lllllll}
\hline variable & $\beta$ & SE & Wald & $P$ value & OR & $95 \%$ CI \\
\hline D-dimer & 0.513 & 0.223 & 5.318 & $0.021^{*}$ & 1.671 & $1.080 \sim 2.584$ \\
C-reactive protein & 0.019 & 0.007 & 6.553 & $0.01^{*}$ & 1.019 & $1.004 \sim 1.034$ \\
lactate dehydrogenase & 0.013 & 0.005 & 7.962 & $0.005^{*}$ & 1.013 & $1.004 \sim 1.023$ \\
white blood cell count & 0.167 & 0.074 & 5.108 & $0.024^{*}$ & 1.181 & $1.022 \sim 1.365$ \\
neutrophil count & 0.220 & 0.086 & 6.577 & $0.01^{*}$ & 1.246 & $1.053 \sim 1.473$ \\
neutrophil percentage & 0.197 & 0.056 & 12.188 & $<0.01^{*}$ & 1.217 & $1.090 \sim 1.359$ \\
lymphocyte count & -2.844 & 1.046 & 7.391 & $0.007^{*}$ & 0.058 & $0.007 \sim 0.452$ \\
lymphocyte percentage & -0.343 & 0.096 & 12.703 & $<0.01^{*}$ & 0.710 & $0.588 \sim 0.857$ \\
\hline
\end{tabular}

$* P<0.05$.

Table 6: Univariate logistic regression analysis of the risk factors for death in COVID-19 patients combined with chronic diseases

\begin{tabular}{lllllll}
\hline variable & $\beta$ & SE & Wald & $P$ value & OR & $95 \%$ CI \\
\hline D-dimer & 0.344 & 0.127 & 7.296 & $0.007 *$ & 1.41 & $1.099 \sim 1.809$ \\
lactate dehydrogenase & 0.009 & 0.003 & 9.581 & $0.002 *$ & 1.009 & $1.003 \sim 1.015$ \\
white blood cell count & 0.128 & 0.064 & 3.974 & $0.046 *$ & 1.137 & $1.002 \sim 1.290$ \\
neutrophil count & 0.159 & 0.071 & 5.039 & $0.025^{*}$ & 1.173 & $1.020 \sim 1.384$ \\
neutrophil percentage & 0.143 & 0.048 & 8.863 & $0.003 *$ & 1.154 & $1.050 \sim 1.267$ \\
lymphocyte count & -3.334 & 1.358 & 6.031 & $0.014 *$ & 0.036 & $0.002 \sim 0.510$ \\
lymphocyte percentage & -0.253 & 0.079 & 10.285 & $0.001 *$ & 0.777 & $0.665 \sim 0.907$ \\
blood urea nitrogen & 0.126 & 0.062 & 4.128 & $0.042 *$ & 1.135 & $1.004 \sim 1.281$ \\
\hline
\end{tabular}

$* P<0.05$.

Table7: multivariate analysis of the risk factors for death in COVID-19 patients combined with chronic diseases

\begin{tabular}{lllllll}
\hline variable & $\beta$ & SE & Wald & $P$ value & OR & 95\% CI \\
\hline lactate dehydrogenase & 0.008 & 0.004 & 4.352 & 0.037 & 1.008 & $1.000 \sim 1.015$ \\
\hline
\end{tabular}


Figure 1. Comorbidity percentage in common pneumonia and severe pneumonia patients was plotted versus each other. Diagonal (gray, dotted) indicated a hypothetically equal percentage between the two groups.

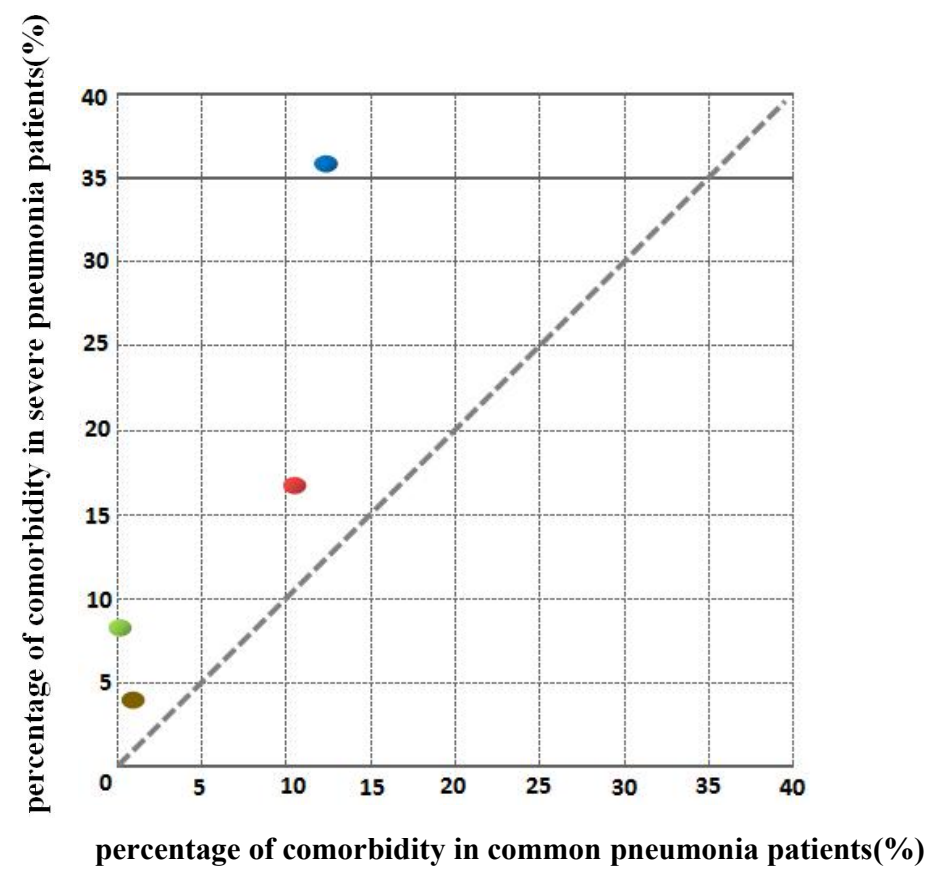

Figure 2. Comorbidity percentage in death and survival group was plotted versus each other. Diagonal (gray, dotted) indicated a hypothetically equal percentage between the two groups.

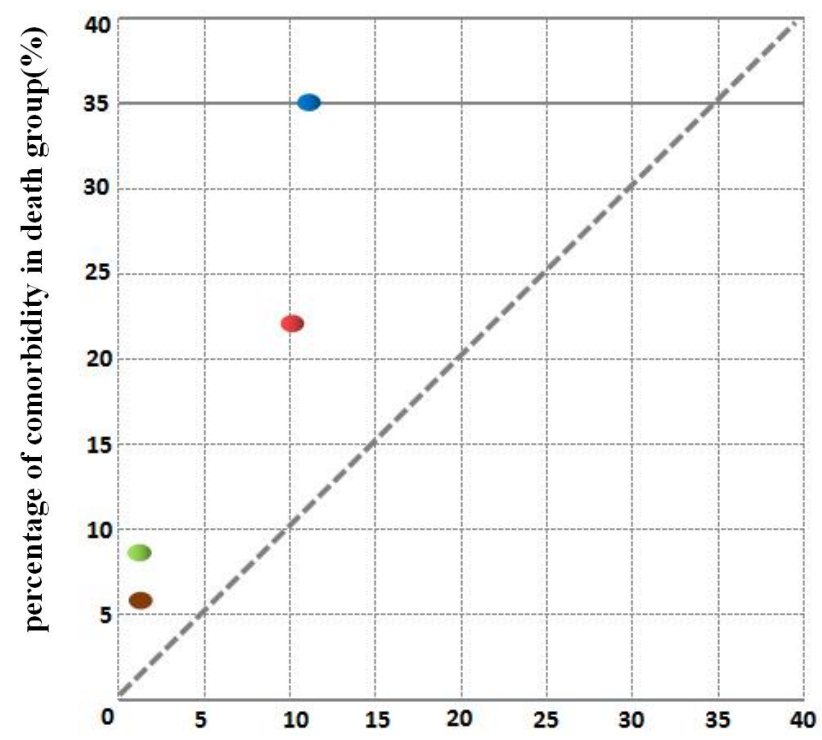

diabetes

- hypertension

coronary

- chronic respiratory system disease

percentage of comorbidity in survival group(\%)

\section{Cox regression analysis and survival curve}

Cox regression analysis showed that compared with simple COVID-19 group, the RR 
$(95 \%$ CI $)$ in COVID-19 combined with chronic diseases group were 2.187 (1.141 4.191) for death $(P<0.05)$. The results of survival analysis revealed that the survival time of patients in the COVID-19 combined with chronic diseases group was significantly shorter than that in the simple COVID-19 group (Fig 4).

Figure 3. The survival curves of the simple COVID-19 group and COVID-19 combined with chronic diseases group

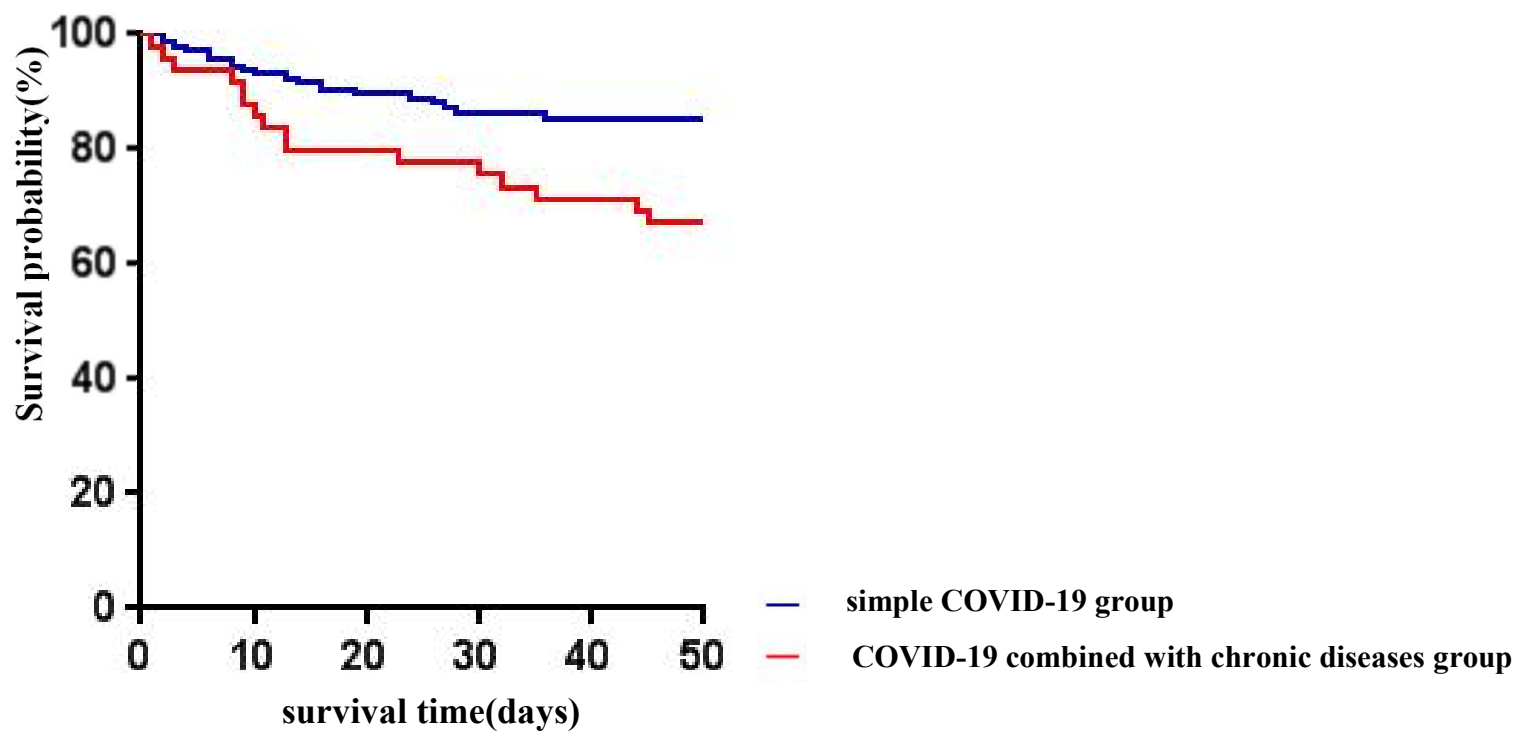

\section{Discussion}

Patients with hypertension, diabetes and coronary heart disease have worse clinical outcomes when infected with coronavirus [11-14]. From previous studies of the fatal cases of severe acute respiratory syndrome coronavirus (SARS-CoV) pneumonia, the comorbidities of hypertension, diabetes and coronary heart disease were found to be dangerous factors that resulted in death [15-16]. Also, a systematic analysis of 637 Middle East respiratory syndrome coronavirus (MERS-CoV) cases suggests that diabetes and hypertension are equally prevalent in approximately $50 \%$ of the patients while coronary heart disease are present in 30\% [17]. However, the mechanisms for high morbidity and mortality of patients with comorbidities are unknown. The SARS-CoV-2, a positive strand RNA virus, has been seen to infect humans through the angiotensin converting enzyme -2 (ACE-2) receptor [18-19]. The ACE-2 receptor is a part of the dual system renin-angiotensin-system (RAS) consisting of 
ACE-Ang-II-AT1R axis and ACE-2-Ang-(1-7)-Mas axis. In metabolic disorders and with increased age, it is known that there is an upregulation of ACE-Ang-II-AT1R axis with a downregulation of ACE-2-Ang-(1-7)-Mas axis. ACE-2-Ang-(1-7)-Mas axis has anti-inflammatory and antifibrotic effects on the respiratory system and anti-inflammatory, antioxidative stress, and protective effects on vascular function, protects against myocardial fibrosis, nephropathy, pancreatitis, and insulin resistance. The already strained ACE-2-Ang-(1-7)-Mas in metabolic disorders is further stressed due to the use of the ACE-2 by the virus for entry, which can affects the prognosis in terms of respiratory compromise[20]. Many scholars believe that this may be one of the possible mechanisms for the high mortality of COVID-19 in patients with underlying metabolic conditions and they believe that ACE-2-based therapy has been proposed as a potential therapeutic approach in COVID-19 pneumonia[21].

There is growing literature exploring that myocardial injury is one of the important pathogenic features of COVID-19[22-25]. In a cohort of 52 critically ill adult patients with SARS-CoV-2 pneumonia who were admitted to the intensive care unit (ICU) of Wuhan Jin Yin-tan hospital, 12 patients (23\%) had cardiac injury which was defined as an elevated serum level of high-sensitivity cardiac troponin I (hs-TnI)[26]. In concert with previous studies, we found that increased cardiac biomarkers mainly myoglobin and cardiac troponins $\mathrm{T}$ in the COVID-19 patients combined with chronic diseases especially those with severe pneumonia or death. The proposed mechanisms of myocardial injury are direct damage to the cardiomyocytes, systemic inflammation, myocardial interstitial fibrosis, interferon mediated immune response, exaggerated cytokine response by Type 1 and 2 helper $\mathrm{T}$ cells, in addition to coronary plaque destabilization, and hypoxia[27-29]. We suggest that myocardial injury is a problem that cannot be ignored in patients with COVID-19. It has also been proposed that in patients with COVID-19 had increased coagulation activity, marked by increased D-dimer concentrations[30]. Study has proved that D-dimer might help in early recognition of these high-risk patients and also predict outcome[31]. We also observed that D-dimer is one of the risk factors for severe pneumonia or death among COVID-19 patients combined with chronic diseases. Contributory mechanisms 
include systemic pro-inflammatory cytokine responses that are mediators of atherosclerosis directly contributing to plaque rupture through local inflammation, induction of procoagulant factors, and haemodynamic changes, which predispose to ischaemia and thrombosis[32]. However, due to the limitations of existing evidence, future research is needed to elaborate on the potential mechanisms.

In this study, lactate dehydrogenase (LDH) were independent risk factors for death among COVID-19 patients combined with chronic diseases. LDH is a cytoplasmic glycolytic enzyme found in all most every tissue. Its elevation generally indicates tissue damage. Raised LDH was a common findings in patients infected with MERS-CoV [33-35]. High LDH levels has previously been shown to be an independent prognostic indicator of SARS infection which can help clinicians to predict adverse clinical outcome [36-38]. It was also reported to be one of the factors tightly associated with mortality of acute respiratory distress syndrome (ARDS) [39]. Our finding of increased LDH in severe pneumonia group and death group indicated the possibility of subclinical tissue damage. As the disease progresses, not only the damage of lung but also involvement in multiple tissues and organs can be observed in severe patients, which indicates systemic organ damage caused by the excessive reaction of the immune response. Thus, we suggest that LDH levels could be used as a surrogate marker help to locate damaged tissues or organs.

Our study has some limitations. First, we no doubt missed patients who were asymptomatic or had mild cases and who were treated at home, so our study cohort may represent the more severe end of Covid-19. Second, some specific information was missing in this study, such as symptoms, chest CT scans, supportive treatment and living status. Third, data generation was clinically driven and not systematic. Last but not least, interpretation of our findings might be limited by the sample size, which may have some impact on the statistical results. Based on the limitations above, a multicenter study will be needed to expand the sample size and to conduct more rigorous randomized controlled trials.

\section{Conclusions:}


In summary, our results indicate that the risk factors for severe pneumonia were D-dimer, C-reactive protein, lactate dehydrogenase, white blood cell count, neutrophil count and neutrophil percentage, whereas the risk factors for death were D-dimer, lactate dehydrogenase, white blood cell count, neutrophil count, neutrophil percentage and blood urea nitrogen among COVID-19 patients combined with diabetes, hypertension or coronary. Lactate dehydrogenase were independent risk factors for death. The mortality rate of COVID-19 patients combined with diabetes, hypertension or coronary was higher than that of simple COVID-19 patients.

\section{Declarations}

\section{Authors' Contributions}

H. L. contributed to literature search and drafting the manuscript. J.L. and J.Z. contributed to data collection and literature search. L.Y., D.W., L.Z. and X.D. contributed to make grammatical revisions and polish the language. G.Y. contributed to the study conception and final approval of the manuscript. G.Y. is the guarantors of this work and, as such, had full access to all data in the study and take responsibility for the integrity of data and the accuracy of the data analysis. Here, we show our sincere respect to all medical staff around the world gathered in an emergency and ran to the front line of the epidemic who put their personal safety aside and fought hard with the sick devil.

\section{Acknowledgements}

All the authors of this manuscript have made substantial contributions to this work.

\section{Competing Interests}

None of the authors have any potential conflict of interests associated with this research.

\section{Availability of data and materials}

The datasets used and/or analysed during the current study are available from the 
authors on request.

\section{Ethics approval and consent to participate}

The study was approved by the Research Ethics Commission of FPHJD (Approval No. 2020021) and written consent was waived by Ethics Commission of FPHJD, and all participants provided written informed consents.

\section{Consent for publication}

All participants provided written informed consents.

\section{Disclosure}

Each of the authors acknowledges that he or she participated sufficiently in the work to take public responsibility for its content.

\section{Funding}

This study was supported in part by grants from the National Natural Science Foundation of China $(81870548,81570721)$, Key research and development projects of social development in Jiangsu Province, China (BE2018692), the Natural Science Foundation of Jiangsu Province, China (BK2019222), the High Caliber Medical Personnel Foundation of Jiangsu Province (LGY2016053), the Six Talent Peaks

Project in Jiangsu Province (2015-WSN-006), the Science and Technology Commission of Zhenjiang City (SH2019041), the “169-Phase V” Science Research Project of Zhenjiang.

\section{Publisher's Note}

Springer Nature remains neutral with regard to jurisdictional claims in published maps and institutional affiliations.

\section{Reference:}


[1] Xu X, Chen P, Wang J, et al. Evolution of the novel coronavirus from the ongoing Wuhan outbreak and modeling of its spike protein for risk of human transmission. Sci China Life Sci. 2020;63(3):457 - 460. doi:10.1007/s11427-020-1637-5

[2] Chang D, Lin M, Wei L, et al. Epidemiologic and Clinical Characteristics of Novel Coronavirus Infections Involving 13 Patients Outside Wuhan, China [published online ahead of print, 2020 Feb 7]. JAMA. 2020;323(11):1092 - 1093. doi:10.1001/jama.2020.1623

[3] Holshue ML, DeBolt C, Lindquist S, et al. First Case of 2019 Novel Coronavirus in the United States. N Engl J Med. 2020;382(10):929 - 936. doi:10.1056/NEJMoa2001191

[4] Chen H, Ai L, Lu H, Li H. Clinical and imaging features of COVID-19 [published online ahead of print, 2020 Apr 27]. Radiol Infect Dis. 2020;10.1016/j.jrid.2020.04.003. doi:10.1016/j.jrid.2020.04.003

[5] Zhou F, Yu T, Du R, et al. Clinical course and risk factors for mortality of adult inpatients with COVID-19 in Wuhan, China: a retrospective cohort study [published correction appears in Lancet. 2020 Mar 28;395(10229):1038] [published correction appears in Lancet. 2020 Mar 28;395(10229):1038]. Lancet. 2020;395(10229):1054 - 1062. doi:10.1016/S0140-6736(20)30566-3

[6] Wang D, Hu B, Hu C, et al. Clinical Characteristics of 138 Hospitalized Patients With 2019 Novel Coronavirus-Infected Pneumonia in Wuhan, China [published online ahead of print, 2020 Feb 7]. JAMA. 2020;323(11):1061-1069. doi:10.1001/jama.2020.1585

[7] Ojha R, Huang D, An H, et al. Distribution of ischemic infarction and stenosis of intra- and extracranial arteries in young Chinese patients with ischemic stroke. BMC Cardiovasc Disord. 2015;15:158. Published 2015 Nov 23. doi:10.1186/s12872-015-0147-5

[8] Chen HX, Wang LJ, Yang Y, Yue FX, Chen LM, Xing YQ. The prevalence of intracranial stenosis in patients at low and moderate risk of stroke. Ther Adv Neurol Disord. 2019;12:1756286419869532. Published 2019 Aug 13. doi:10.1177/1756286419869532

[9] Arbab-Zadeh A, Fuster V. The Risk Continuum of Atherosclerosis and its Implications for Defining CHD by Coronary Angiography. J Am Coll Cardiol. 2016;68(22):2467 - 2478. doi:10.1016/j.jacc.2016.08.069

[10] National Health Commission of the People's Republic of China. Chinese management guideline for COVID-19 (version 6.0). Feb 19, 2020. http://www.nhc.gov.cn/yzygj/s7653p/202002/8334a832

6dd94d329df351d7da8aefc2/files/b218cfeb1bc54639af227f922bf6b817. pdf (accessed Feb 19, 2020; in Chinese).

[11] Ji HL, Zhao R, Matalon S, Matthay MA. Elevated Plasmin(ogen) as a Common Risk Factor for COVID-19 Susceptibility. Physiol Rev. 2020;100(3):1065 - 1075. doi:10.1152/physrev.00013.2020

[12] Zhou F, Yu T, Du R, et al. Clinical course and risk factors for mortality of adult inpatients with COVID-19 in Wuhan, China: a retrospective cohort study [published correction appears in Lancet. 2020 Mar 28;395(10229):1038] [published correction 
appears in Lancet. 2020 Mar 28;395(10229):1038]. Lancet. 2020;395(10229):1054 - 1062.

[13] Wang D, Hu B, Hu C, et al. Clinical Characteristics of 138 Hospitalized Patients With 2019 Novel Coronavirus-Infected Pneumonia in Wuhan, China [published online ahead of print, 2020 Feb 7]. JAMA. 2020;323(11):1061-1069. doi:10.1001/jama.2020.1585

[14] Wu C, Chen X, Cai Y, et al. Risk Factors Associated With Acute Respiratory Distress Syndrome and Death in Patients With Coronavirus Disease 2019 Pneumonia in Wuhan, China [published online ahead of print, 2020 Mar 13]. JAMA Intern Med. 2020;e200994. doi:10.1001/jamainternmed.2020.0994

[15] Chan JW, Ng CK, Chan YH, et al. Short term outcome and risk factors for adverse clinical outcomes in adults with severe acute respiratory syndrome (SARS). Thorax. 2003;58(8):686 - 689. doi:10.1136/thorax.58.8.686

[16] Booth CM, Matukas LM, Tomlinson GA, et al. Clinical features and short-term outcomes of 144 patients with SARS in the greater Toronto area [published correction appears in JAMA. 2003 Jul 16;290(3):334]. JAMA. 2003;289(21):2801 - 2809. doi:10.1001/jama.289.21.JOC30885

[17] Badawi A, Ryoo SG. Prevalence of comorbidities in the Middle East respiratory syndrome coronavirus (MERS-CoV): a systematic review and meta-analysis. Int $\mathrm{J}$ Infect Dis. 2016;49:129 - 133. doi:10.1016/j.ijid.2016.06.015

[18] Hoffmann M, Kleine-Weber H, Schroeder S, et al. SARS-CoV-2 Cell Entry Depends on ACE2 and TMPRSS2 and Is Blocked by a Clinically Proven Protease Inhibitor. Cell. 2020;181(2):271 - 280.e8. doi:10.1016/j.cell.2020.02.052

[19] Yan R, Zhang Y, Li Y, Xia L, Guo Y, Zhou Q. Structural basis for the recognition of SARS-CoV-2 by full-length human ACE2. Science. 2020;367(6485):1444 - 1448. doi:10.1126/science.abb2762

[20] Dalan R, Bornstein SR, El-Armouche A, et al. The ACE-2 in COVID-19: Foe or Friend?. Horm Metab Res. 2020;52(5):257 - 263. doi:10.1055/a-1155-0501

[21] Zhang H, Penninger JM, Li Y, Zhong N, Slutsky AS. Angiotensin-converting enzyme 2 (ACE2) as a SARS-CoV-2 receptor: molecular mechanisms and potential therapeutic target. Intensive Care Med. 2020;46(4):586 - 590. doi:10.1007/s00134-020-05985-9

[22] Zhou F, Yu T, Du R, et al. Clinical course and risk factors for mortality of adult inpatients with COVID-19 in Wuhan, China: a retrospective cohort study [published correction appears in Lancet. 2020 Mar 28;395(10229):1038] [published correction appears in Lancet. 2020 Mar 28;395(10229):1038]. Lancet. 2020;395(10229):1054 - 1062. doi:10.1016/S0140-6736(20)30566-3

[23] Guan WJ, Ni ZY, Hu Y, et al. Clinical Characteristics of Coronavirus Disease 2019 in China. N Engl J Med. 2020;382(18):1708 - 1720. doi:10.1056/NEJMoa2002032

[24] Musa S. Hepatic and gastrointestinal involvement in coronavirus disease 2019 (COVID-19): What do we know till now?. Arab J Gastroenterol. 2020;21(1):3 - 8. doi:10.1016/j.ajg.2020.03.002 
[25] Madjid M, Safavi-Naeini P, Solomon SD, Vardeny O. Potential Effects of Coronaviruses on the Cardiovascular System: A Review [published online ahead of print, 2020 Mar 27]. JAMA Cardiol. 2020;10.1001/jamacardio.2020.1286. doi:10.1001/jamacardio.2020.1286

[26] Yang X, Yu Y, Xu J, et al. Clinical course and outcomes of critically ill patients with SARS-CoV-2 pneumonia in Wuhan, China: a single-centered, retrospective, observational study [published correction appears in Lancet Respir Med. 2020 Apr;8(4):e26]. Lancet Respir Med. 2020;8(5):475 - 481. doi:10.1016/S2213-2600(20)30079-5

[27] Babapoor-Farrokhran S, Gill D, Walker J, Rasekhi RT, Bozorgnia B, Amanullah A. Myocardial injury and COVID-19: Possible mechanisms. Life Sci. 2020;253:117723. doi:10.1016/j.lfs.2020.117723

[28] Zhao X, Nicholls JM, Chen YG. Severe acute respiratory syndrome-associated coronavirus nucleocapsid protein interacts with Smad3 and modulates transforming growth factor-beta signaling. J Biol Chem. 2008;283(6):3272 - 3280 . doi:10.1074/jbc.M708033200

[29] Cameron MJ, Bermejo-Martin JF, Danesh A, Muller MP, Kelvin DJ. Human immunopathogenesis of severe acute respiratory syndrome (SARS). Virus Res. 2008;133(1):13 - 19. doi:10.1016/j.virusres.2007.02.014

[30] Milbrandt EB, Reade MC, Lee M, et al. Prevalence and significance of coagulation abnormalities in community-acquired pneumonia. Mol Med. 2009;15(11-12):438 - 445. doi:10.2119/molmed.2009.00091

[31] Kollias A, Kyriakoulis KG, Dimakakos E, Poulakou G, Stergiou GS, Syrigos K. Thromboembolic risk and anticoagulant therapy in COVID-19 patients: emerging evidence and call for action. $\mathrm{Br} \mathrm{J}$ Haematol. 2020;189(5):846 - 847. doi:10.1111/bjh.16727

[32] Smeeth L, Thomas SL, Hall AJ, Hubbard R, Farrington P, Vallance P. Risk of myocardial infarction and stroke after acute infection or vaccination. N Engl J Med. 2004;351(25):2611 - 2618. doi:10.1056/NEJMoa041747

[33] Assiri A, Al-Tawfiq JA, Al-Rabeeah AA, et al. Epidemiological, demographic, and clinical characteristics of 47 cases of Middle East respiratory syndrome coronavirus disease from Saudi Arabia: a descriptive study. Lancet Infect Dis. 2013;13(9):752 - 761. doi:10.1016/S1473-3099(13)70204-4

[34] Alsolamy S. Middle East respiratory syndrome: knowledge to date. Crit Care Med. 2015;43(6):1283 - 1290. doi:10.1097/CCM.0000000000000966

[35] Al Ghamdi M, Alghamdi KM, Ghandoora Y, et al. Treatment outcomes for patients with Middle Eastern Respiratory Syndrome Coronavirus (MERS CoV) infection at a coronavirus referral center in the Kingdom of Saudi Arabia. BMC Infect Dis. 2016;16:174. Published 2016 Apr 21. doi:10.1186/s12879-016-1492-4

[36] Lesens O, Hustache-Mathieu L, Hansmann Y, Remy V, Hoen B, Christmann D. Syndrome respiratoire aigu sévère (Sras) Les questions posées par la prise en charge d'un patient à Besançon et Strasbourg [Severe acute respiratory syndrome (SARS). The questions raised by the management of a patient in Besançon and Strasbourg]. Presse Med. 2003;32(29):1359 - 1364. 
[37] Chen CY, Lee CH, Liu CY, Wang JH, Wang LM, Perng RP. Clinical features and outcomes of severe acute respiratory syndrome and predictive factors for acute respiratory distress syndrome. J Chin Med Assoc. 2005;68(1):4 - 10. doi:10.1016/S1726-4901(09)70124-8

[38] Lee $\mathrm{N}$, Hui $\mathrm{D}, \mathrm{Wu} \mathrm{A}$, et al. A major outbreak of severe acute respiratory syndrome in Hong Kong. N Engl J Med. 2003;348(20):1986 - 1994. doi:10.1056/NEJMoa030685

[39] Hoeboer SH, Oudemans-van Straaten HM, Groeneveld AB. Albumin rather than C-reactive protein may be valuable in predicting and monitoring the severity and course of acute respiratory distress syndrome in critically ill patients with or at risk for the syndrome after new onset fever. BMC Pulm Med. 2015;15:22. Published 2015 Mar 14. doi:10.1186/s12890-015-0015-1 
Figures

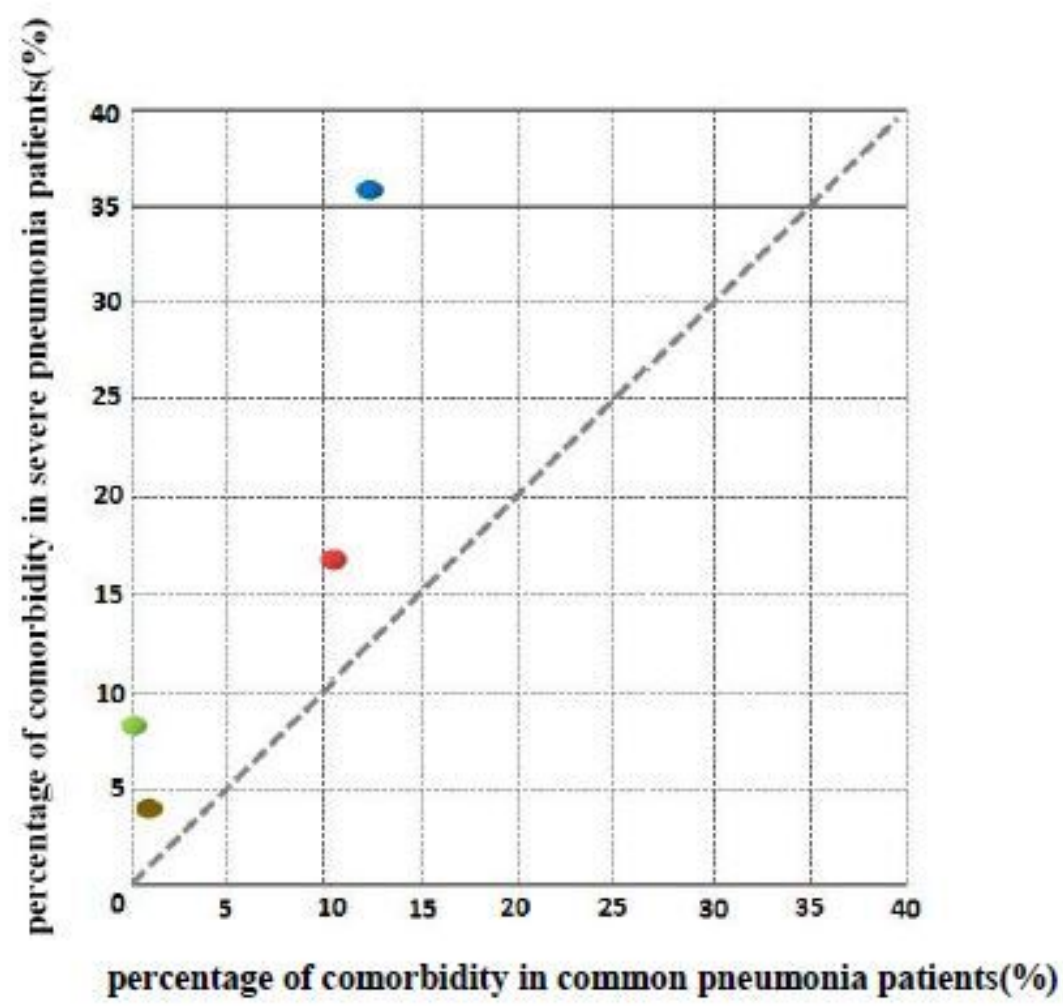

\section{Figure 1}

Comorbidity percentage in common pneumonia and severe pneumonia patients was plotted versus each other. Diagonal (gray, dotted) indicated a hypothetically equal percentage between the two groups.

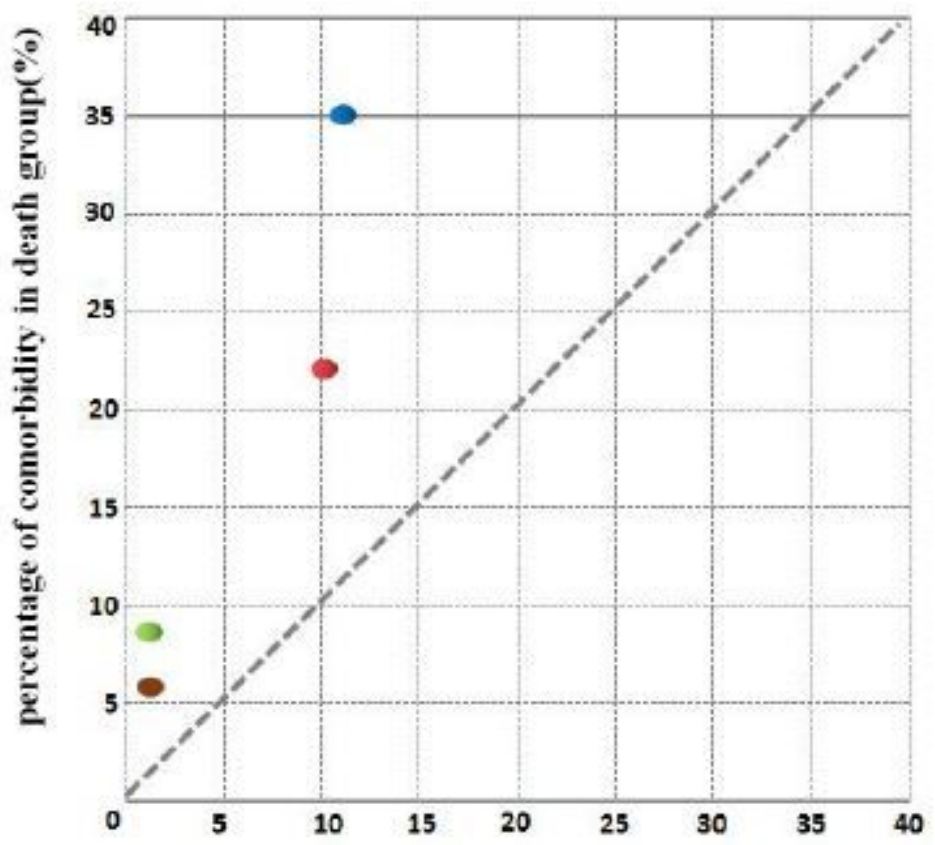

diabetes

- hypertension

coronary

- chronic respiratory system disease

percentage of comorbidity in survival group $(\%)$ 
Figure 2

Comorbidity percentage in death and survival group was plotted versus each other. Diagonal (gray, dotted) indicated a hypothetically equal percentage between the two groups.

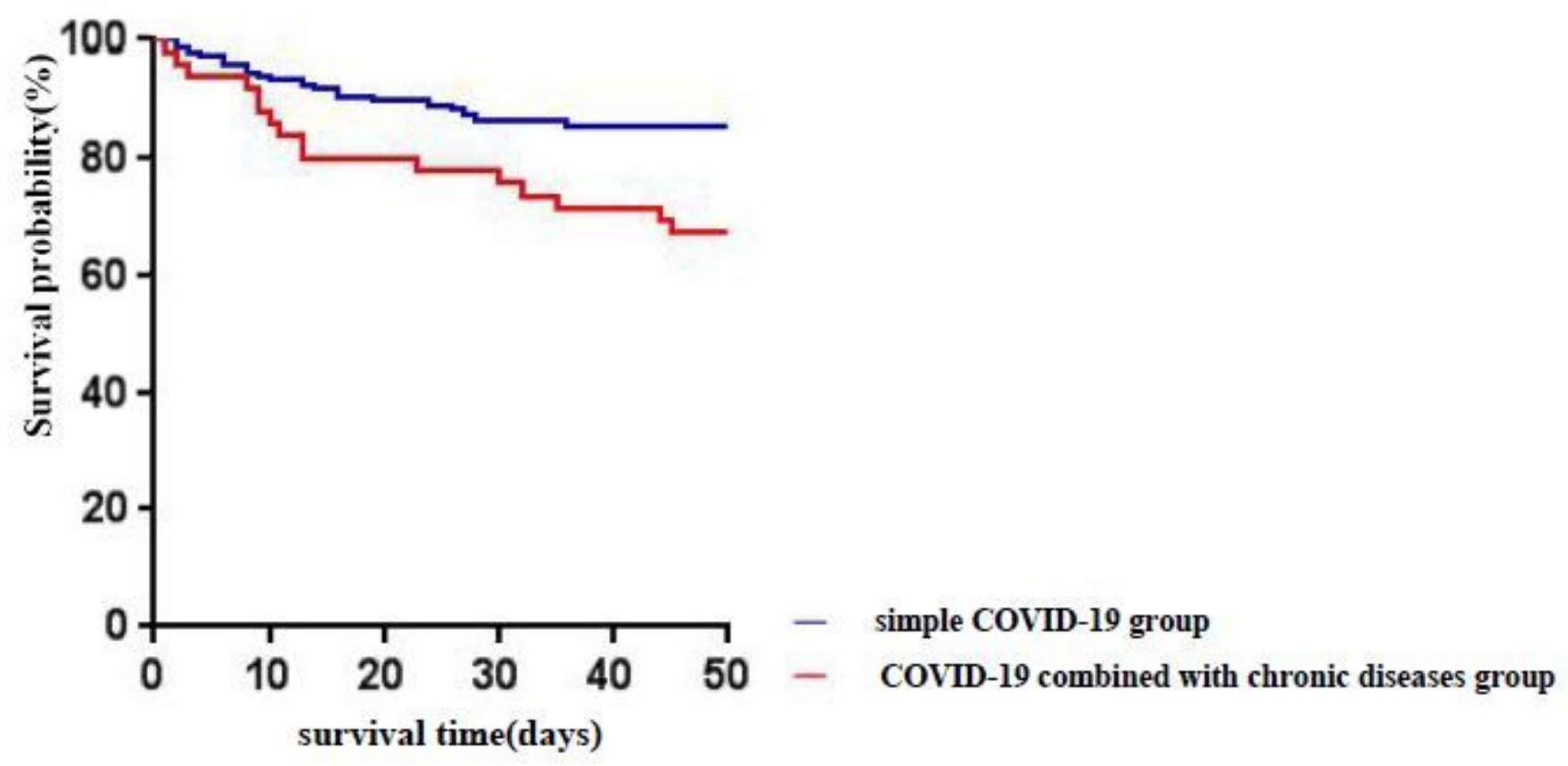

Figure 3

The survival curves of the simple COVID-19 group and COVID-19 combined with chronic diseases group 